\title{
Temperature effects for isothermal polymer crystallization kinetics
}

\author{
Jiao Yang and Benjamin J. McCoy \\ Department of Chemical Engineering, Louisiana State University, Baton Rouge, Louisiana 70803 \\ Giridhar Madras ${ }^{\text {a) }}$ \\ Department of Chemical Engineering, Indian Institute of Science, Bangalore 560 012, India
}

\begin{abstract}
We adopt the cluster size distribution model to investigate the effect of temperature on homogeneous nucleation and crystal growth for isothermal polymer crystallization. The model includes the temperature effects of interfacial energy, nucleation rate, growth and dissociation rate coefficients, and equilibrium solubility. The time dependencies of polymer concentration, number and size of crystals, and crystallinity (in Avrami plots) are presented for different temperatures. The denucleation (Ostwald ripening effect) is also investigated by comparing moment and numerical solutions of the population balance equations. Agreement between the model results and temperature-sensitive experimental measurements for different polymer systems required strong temperature dependence for the crystal-melt interfacial energy.
\end{abstract}

\section{INTRODUCTION}

Polymer crystallization is a complex phenomenon in materials and pharmaceutical processing that profoundly affects microscopic structure and properties of polymer products. Understanding the kinetics of polymer crystallization is a significant scientific challenge. The nucleation and growth of polymer crystals involve the dynamics of polymer chains, including the formation of folded chain structures. To explore the kinetics of polymer crystallization, we recently adopted a cluster size-distribution kinetics model $^{1}$ for nucleation accompanied by crystal growth and Ostwald ripening. Population balance equations based on crystal size distribution and concentration of amorphous polymer segments were established and the related dynamic moment equations were also developed. The model accounted for heterogeneous or homogeneous nucleation and crystal growth. The results were graphed as Avrami plots, thus providing a fundamental basis for this common method of crystallization analysis. In the current paper, we will focus on temperature effects of polymer crystallization. Frequently overlapping with crystal growth, ${ }^{2,3}$ nucleation initiates the phase transition from fluid (liquid) phase to solid (crystal) phase. Among other features, this overlap complicates the kinetics and dynamics of such systems. The effect of temperature on interfacial energy, growth rate coefficients, and equilibrium solubility can potentially be used to design manufacturing methods and to control product properties, e.g., average size of polymer crystal, microscopic structure, and polydispersity of crystal size.

Much research effort has been directed toward polymer crystallization, but a detailed understanding of temperature effects is still lacking. Recent experimental studies ${ }^{4-9}$ for iso-

\footnotetext{
${ }^{\text {a) }}$ Author to whom correspondence should be addressed. Telephone: 91-0802293-2321. Fax: 91-080-2360-0683. Electronic mail: giridhar@chemeng.iisc.ernet.in
}

thermal crystallization of different polymers reported that small changes in temperature caused substantial changes in the crystallization rate. The characteristic Avrami exponents (slopes of Avrami plots) varied from 1 to 4 and were independent of temperature. Most of the data also show a deviation from straight-line Avrami plots at long times (large crystallinity) by curving toward a constant asymptote. Classical nucleation theory based on estimates of the interfacial free energy was applied for nucleation between $T_{g}$ (glass transition temperature) and $T_{m}$ (melting temperature) and showed that the crystallization kinetics were sensitive to small temperature changes. ${ }^{10}$ A study of the molecular weight effect for isothermal crystallization indicated that the crystallization rate is essentially not affected by the polymer molecular weight. ${ }^{11}$ A challenge in modeling temperature effects for polymer crystallization is to represent quantitatively these effects, and in particular the strong influence of temperature on crystallinity.

Crystal growth temperature dependence is controlled by the competition among kinetic and thermodynamic phenomena. ${ }^{12}$ High temperature favors a large crystal growth rate but also enhanced crystal solubility. The nucleation rate temperature dependence complicates the temperature effect even more. For the polymer melt, nucleation occurs when the temperature drops below the melting temperature $T_{m}$. By enhancing the equilibrium solubility, increasing temperature decreases supersaturation. Furthermore, interfacial energy and thus nucleation vanish at $T_{m}$. Decreasing temperature to the glass temperature $T_{g}$, however, also causes nucleation to vanish or become infinitesimal because of the drastic increase of fluid viscosity. Therefore, nucleation for polymer melt is limited to the temperature range ${ }^{13}$ between $T_{g}$ and $T_{m}$, and a maximum nucleation rate is expected between these two temperatures.

Crystallites in polymers exert a major influence on their bulk properties. Modification of crystallinity can signifi- 
cantly alter mechanical and optical properties. Thus, the thermal history of a polymer, in particular between $T_{g}$ and $T_{m}$, greatly influences its end-use value. ${ }^{14}$ Understanding the temperature effect, the key parameter influencing industrial crystallization, is essential for rational manufacturing design and operation. Potentially one can exploit the competition between kinetics and thermodynamic driving forces to manipulate the crystallization rate, crystal size, and particle polydispersity. Our approach is to represent the dynamics and kinetics of nucleation and growth processes by a cluster size-distribution model. The temperature effect is incorporated into the model by considering the temperature dependence of interfacial energy, equilibrium solubility, and growth rate coefficient. We begin in the next section by reviewing the theory of nucleation, growth, and coarsening for polymer crystallization. In a following section, quantitative results of the theory are presented and discussed, along with comparison with published experimental data. Conclusions are presented last.

\section{THEORY}

Temperature influences nucleation and crystal growth in polymer processing through kinetics and thermodynamics. As polymer melt or solution is cooled, the molecule tends to move toward the lowest energy conformation, leading to the formation of ordered chains. ${ }^{15}$ Chain entanglement, however, impedes the ordering required for nucleation. As the polymer solution or melt is cooled, diffusion coefficients decrease. When cooled to the glass transition temperature $T_{g}$, the polymer solution or melt becomes glassy and nucleation is totally quenched because no molecules are able to overcome the diffusion resistance to aggregate and align into a nucleus. The growth of the crystalline region is also impeded by reduced diffusion at low temperature and thermal redispersion of the chains at the crystal/melt surface at higher temperature.

Temperature effects on nucleation rate, according to the classic nucleation theory, ${ }^{16}$ originate from the interfacial energy difference and equilibrium solubility change at various temperatures. Temperature influences incorporated in crystal growth rate include the prefactor of growth rate coefficient, the Gibbs-Thomson effect of particle curvature, ${ }^{17}$ and the phase transition energy (heat of solidification). The dissociation rate coefficient is related to the growth rate coefficient by microscopic reversibility, thereby determining its temperature dependence.

For a two-dimensional (2D) equilateral lamellar crystal, according to classical homogeneous nucleation theory, the sum of surface energy and formation free energy can be represented as ${ }^{1,18}$

$$
W(a)=4 a b \sigma-a^{2} b\left(\rho / x_{m}\right) R T \ln S_{\mathrm{sat}},
$$

where $-R T \ln S_{\text {sat }}$ is the chemical potential difference between the two phases in terms of the supersaturation, $S_{\text {sat }}$, the ratio of bulk concentration $m^{(0)}$ over equilibrium concentration $m_{\mathrm{eq}}^{(0)}$. Also, $\sigma$ is the crystal interfacial energy, $\rho$ is the density of the cluster, $x_{m}$ is the molecular weight of the monomer, $a$ is the lateral length, and $b$ is the thickness of the lamellae. The energy $W(a)$ of a cluster increases with $a$ and then decreases from the maximum value $W^{*}$ at the critical lamellar length,

$$
a^{*}=2 \sigma x_{m} /\left(\rho R T \ln S_{\mathrm{sat}}\right) \text {. }
$$

According to the similarity of thermodynamic driving-force expressions for nucleation from polymer solution and polymer melt, ${ }^{19-21}$ the supersaturation $S_{\text {sat }}$ can be written in terms of number of free macromolecules $m^{(0)}$ and $m_{\mathrm{eq}}^{(0)}, S_{\text {sat }}$ $=m^{(0)} / m_{\text {eq }}^{(0)}$, instead of melting enthalpy and temperature driving force. Thus, the maximum energy of the cluster is represented as

$$
W^{*}=4 x_{m} b \sigma^{2} / \rho R T\left[\ln \left(m^{(0)} / m_{\mathrm{eq}}^{(0)}\right)\right] .
$$

The nucleation rate, according to classical homogeneous nucleation theory, is the flux over the maximum energy barrier (at $a=a^{*}$ ),

$$
I=k_{n} \exp \left(-W^{*} / R T\right),
$$

with prefactor ${ }^{17}$

$$
k_{n}=\left(m^{(0)}\right)^{2}\left(2 \sigma x_{m} / \pi\right)^{1 / 2} \rho^{-1} .
$$

Thermodynamics provides the temperature dependence of solubility for the corresponding flat surface, $m_{\infty}^{(0)}$ $=\mu_{\infty} \exp (-\Delta H / R T)$, where $\Delta H$ represents the molar energy of phase transition between solid (crystal) phase and liquid phase, and $\mu_{\infty}$ stands for the flat-surface polymer solubility at high temperature.

The temperature dependence of interfacial energy $\sigma$ is approximated by ${ }^{22}$

$$
\sigma=\sigma_{0}\left(1-T / T_{m}\right)^{n}
$$

causing interfacial energy $\sigma$ to vanish at reference temperature $T_{m}$. For $n=0$ or $1, \sigma$ is constant or linearly decreasing, respectively. $^{22}$ But for $n \approx 20, \sigma$ falls sharply for $T \ll T_{m}$, and then decreases gradually to zero as $T$ approaches $T_{m}$. For the present model, this dependence of interfacial energy on $T$ is critical for explaining the temperature dependence of polymer crystallinity.

Similar to cluster growth in the kinetics model of McCoy, ${ }^{23}$ crystal growth can be considered as the gradual building up of polymer molecules on the surface of the nucleus in a melt or solution. A general representation of the reversible deposition of chain segments on the crystal surface is

$$
\mathbf{C}(x)+\mathbf{M}\left(x_{m}\right) \underset{k_{d}(x)}{\stackrel{k_{g}(x)}{\rightleftharpoons}} \mathbf{C}\left(x+x_{m}\right),
$$

where $\mathbf{C}(x)$ represents the cluster of mass $x$ and $\mathbf{M}\left(x_{m}\right)$ is the polymer of uniform molecular weight $x_{m}$ with a distribution written as $m(x, t)=m^{(0)} \delta\left(x-x_{m}\right)$. The mass-dependent rate coefficients $k_{g}(x)$ and $k_{d}(x)$ are for cluster growth and dissociation, respectively. An activation energy for the growth coefficient accounts for its temperature dependence,

$$
k_{g}(x)=\gamma x^{\lambda} \exp (-E / R T) .
$$

The exponent $\lambda$ can be set equal to $0,1 / 3$, and $2 / 3$, representing surface independent, diffusion controlled, and surface dependent growth rate. ${ }^{18}$ A number larger than $2 / 3$ is 
also occasionally suggested, ${ }^{1,24}$ for the increasing mass dependence of deposition rate caused by microstructure changes or shear force during fluid movement. The rate coefficient for dissociation is simply related to $k_{g}(x)$ by microscopic reversibility,

$$
k_{d}(x)=m_{\mathrm{eq}}^{(0)} k_{g}(x) .
$$

For a lamellar crystal with flat growth front, the GibbsThomson effect vanishes, so that the difference between local equilibrium interfacial concentration $m_{\mathrm{eq}}^{(0)}$ and solubility $m_{\infty}^{(0)}$ for a flat surface vanishes, thus $m_{\mathrm{eq}}^{(0)}=m_{\infty}^{(0)}$. The population balance equations that govern the distributions of crystals and macromolecules are ${ }^{25}$

$$
\begin{aligned}
\partial c(x, t) / \partial t= & -k_{d}(x) c(x, t)+\int_{x}^{\infty} k_{d}\left(x^{\prime}\right) c\left(x^{\prime}, t\right) \delta\left(x-\left(x^{\prime}\right.\right. \\
& \left.\left.-x_{m}\right)\right) d x^{\prime}-k_{g}(x) c(x, t) m^{(0)} \int_{0}^{\infty} \delta\left(x^{\prime}-x_{m}\right) d x^{\prime} \\
& +m^{(0)} \int_{0}^{x} k_{g}(x) c\left(x^{\prime}, t\right) \delta\left(x-x_{m}\right) d x^{\prime} \\
& +I \delta\left(x-x^{*}\right),
\end{aligned}
$$

and

$$
\begin{aligned}
\partial m(x, t) / \partial t= & m^{(0)}(t) \int_{0}^{\infty} k_{g}\left(x^{\prime}\right) c\left(x^{\prime}, t\right) d x^{\prime} \\
& +\int_{x}^{\infty} k_{d}\left(x^{\prime}\right) c\left(x^{\prime}, t\right) \delta\left(x-x_{m}\right) d x^{\prime} \\
& -I \delta\left(x-x^{*}\right) x^{*} / x_{m} .
\end{aligned}
$$

Consistent with the definition of rate coefficients in Eq. (7), the population balance equations are based on concentration units of mole/volume. We define dimensionless quantities as follows: ${ }^{26}$

$$
\begin{aligned}
& C=c x_{m} / \mu_{\infty}, \quad c^{(n)}=c^{(n)} / \mu_{\infty} x_{m}^{n}, \quad \xi=x / x_{m}, \\
& \theta=t \gamma \mu_{\infty} x_{m}^{\lambda}, \quad S=m^{(0)} / \mu_{\infty}, \\
& \Theta=T / T_{m}, \quad w=2 \sigma_{0}\left(x_{m} b / \rho\right)^{1 / 2} / R T_{m}, \quad J=I / \gamma \mu_{\infty}^{2} x_{m}^{\lambda}, \\
& \varepsilon=E / R T_{m}, \quad h=\Delta H / R T_{m},
\end{aligned}
$$

where numbers of polymer molecules and polymer crystals, $S$ and $C^{(0)}$, are scaled by solubility at high temperature $\mu_{\infty}$. The supersaturation, $S_{\mathrm{sat}}=m^{(0)} / m_{\mathrm{eq}}^{(0)}=S \exp (h / \Theta)$, evolves to unity at thermodynamic equilibrium. The temperature is scaled by a reference temperature $T_{m}$, the melting temperature of polymer crystal. The interfacial energy, $\omega=w(1$ $-\Theta)^{n} / \Theta$, is expressed in terms of scaled temperature $\Theta$ and the temperature-independent parameter $w$. With the definition of the $n$th moment, $C^{(n)}=\int_{0}^{\infty} x^{n} C(x, \theta) d x$, for the special case $\lambda=0$ the dimensionless population balance equations for crystal growth and nucleation can be represented as moment equations,

$$
d S(\theta) / d \theta=\exp (-\varepsilon / \Theta)[-S(\theta)+\exp (-h / \Theta)] C^{(0)}+J \xi^{*}
$$

and

$d C^{(0)}(\theta) / d \theta=J$,

$d C^{(1)}(\theta) / d \theta=-\exp (-\varepsilon / \Theta)[-S(\theta)+\exp (-h / \Theta)] C^{(0)}-J \xi^{*}$.

Here $J$ represents the scaled homogeneous nucleation rate of crystal of critical mass size $\xi^{*}$ and is a source term in Eqs. (13)-(15). The number of macromolecules in the critical crystal nucleus is

$$
\xi^{*}=\left[w(1-\Theta)^{n} / \Theta(\ln S+h / \Theta)\right]^{2},
$$

which varies with time, because of the time dependence of the scaled number of crystallizing polymers $S$, and is strongly dependent on temperature. The initial conditions are $S(\theta=0)=S_{0}$ and $C^{(0)}(\theta=0)=0$ for homogeneous nucleation. For a batch system without agitation, crystallizing temperature is the only operating condition. In terms of the initial number of polymer molecules $S_{0}$, the scaled mass conservation for a closed system follows from the population balance equations, Eqs. (13) and (15),

$$
C^{(1)}(\theta)+S(\theta)=C_{0}^{(1)}+S_{0},
$$

where $C_{0}^{(1)}$ is the initial cluster mass, representing heterogeneous nuclei and seeds, and is zero for homogeneous nucleation, and $S_{0}$ is the initial number of polymer molecules. At thermodynamic equilibrium, supersaturation $S_{\text {sat }}$ equals unity, so that the thermodynamic driving forces for crystal growth and nucleation rate vanish. Thus the time evolution rates of moments are zero, $d S(\theta) / d \theta=0$ and $d C^{(0)}(\theta) / d \theta=0$. The degree of crystallinity, $X$, is defined as the ratio of the mass crystallized at time $t$ divided by the total mass crystallized,

$$
X=\left(C^{(1)}-C_{0}^{(1)}\right) /\left(C_{\mathrm{eq}}^{(1)}-C_{0}^{(1)}\right) .
$$

Substitution of the mass conservation equation, Eq. (17), simplifies Eq. (18) to

$$
X=\left(S_{0}-S(\theta)\right) /\left(S_{0}-e^{-h / \Theta}\right) \approx 1-S / S_{0},
$$

where at equilibrium, consistent with Eq. (13), $S_{\text {sat }}=1$ and $S=e^{-h / \Theta} \approx 0$, for $h \gg \Theta$. By defining the temperatureindependent prefactor,

$$
J_{0}=\left(2 \sigma_{0} / \pi\right)^{1 / 2} / \rho \gamma x_{m}^{\lambda-1 / 2},
$$

the nucleation rate can be written as

$$
J=J_{0}(1-\Theta)^{n / 2} S^{2} \exp \left[-\left(w(1-\Theta)^{n} / \Theta\right)^{2} /(\ln S+h / \Theta)\right] .
$$

The growth and dissociation rate coefficients are assumed to be mass independent $(\lambda=0)$ in the above moment method. However, these rate coefficients are not always mass independent, especially for polymer solutions with high viscosity. A more general expression for the mass dependence is 

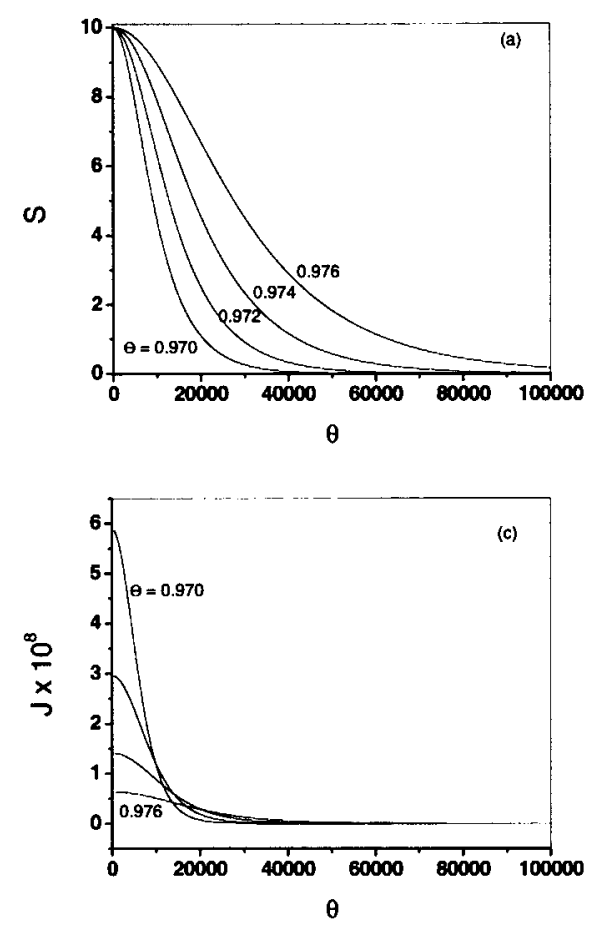
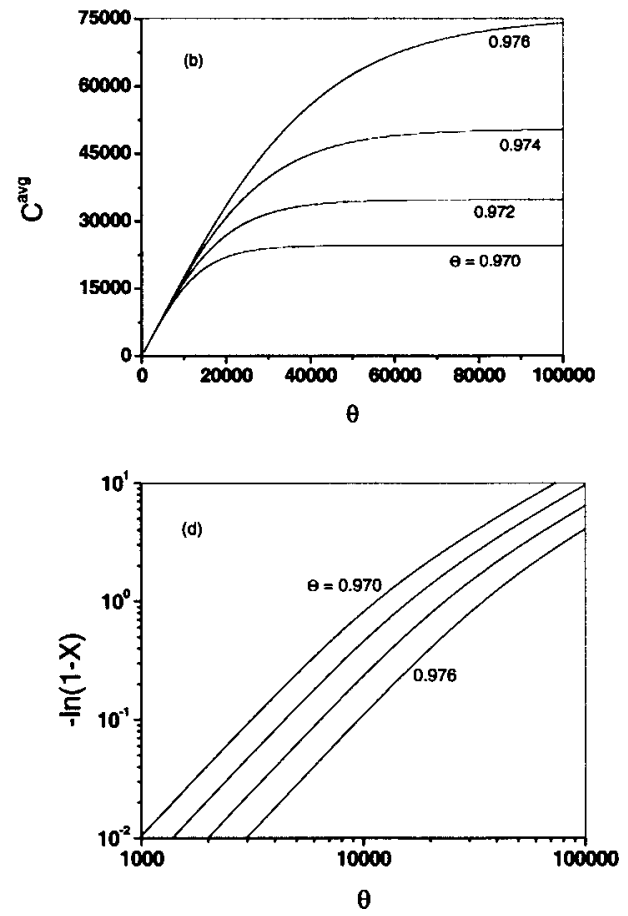

FIG. 1. Time evolution of (a) number of macromolecules $S$, (b) average cluster size $C^{\text {avg }}$, (c) nucleation rate $J$, and (d) $-\ln (1-X)$ at $\Theta=0.970, \quad 0.972$, 0.974 , and 0.976 with parameters given in Table I. the power law ${ }^{18}$ dependence in Eq. (8). With the dimensionless quantities of Eq. (12), Eqs. (10) and (11) can be written as

$$
d S(\theta) / d \theta=\exp (-\varepsilon / \Theta)[-S(\theta)+\exp (-h / \Theta)] C^{(\lambda)}+J \xi^{*}
$$

and

$$
\begin{aligned}
\partial C(\xi, \theta) / \partial \theta= & S(\theta) \exp (-\varepsilon / \Theta)\left(-\xi^{\lambda} C(\xi, \theta)+(\xi-1)^{\lambda} C(\xi\right. \\
& -1, \theta))-\xi^{\lambda} \exp (-(\varepsilon+h) / \Theta) C(\xi, \theta) \\
& +(\xi+1)^{\lambda} \exp (-(\varepsilon+h) / \Theta) C(\xi+1, \theta) \\
& -J \delta\left(\xi-\xi^{*}\right) .
\end{aligned}
$$

Here, $\partial C(\xi, \theta) / \partial \theta$ represents the generation rate of clusters with $\xi$ polymers. Numerical schemes are employed to solve the equations.

\section{RESULTS}

To grasp the temperature dependence of the whole process of crystallization, it is important to understand the temperature dependence of the two overlapping processes: ${ }^{2}$ nucleation and crystal growth. Temperature influences nucleation rate, according to Eq. (21), mainly through supersaturation, $S_{\text {sat }}=S \exp (h / \Theta)$, and interfacial energy, $\sigma=\sigma_{0}(1$ $-\Theta)^{n}$. The nucleation rate decreases near the melting temperature because of the substantial temperature dependence of interfacial energy there. Crystal growth rate, influenced by temperature mainly through the growth activation energy $\varepsilon$ according to Eq. (8), increases with temperature. We are initially concerned with the effect of temperature on the time evolution of crystal size distribution. Figure 1 presents the temperature effects on number of macromolecules, crystal number, crystal size, and nucleation rate at different temperatures close to the reference temperature $T_{m}$. Based on experi- mental measurements, ${ }^{27}$ reasonable parameter values are chosen, as listed in Table I. The mass dependence of growth rate coefficient $\lambda$ is chosen to be 0 , thus the moment equations (13)-(15) are applied to describe nucleation and crystal growth. Considering the sensitivity of crystallinity on temperature, the scaled temperature values $\Theta$ are chosen to be $0.970,0.972,0.974$, and 0.976 . Figure 1 (a) shows the time evolution of polymer concentration, with a larger decrease speed of $S$ at the lower temperature, $\Theta=0.97$. Decreasing temperature speeds up nucleation, and the crystal growth rate decreases slightly because of the lower temperature sensitivity for crystal growth. The time evolution of crystallized mass concentration, not presented in this paper, is simply a mirror image of $S$ according to Eqs. (13) and (15).

The temperature effect on average crystal size is presented in Fig. 1(b). The average crystal size increases with time by crystal growth and later does not change very much as equilibrium is approached. Increasing temperature also increases the average crystal size. According to the definition, $C^{\text {avg }}=C^{(1)} / C^{(0)}$, the decline of average crystal size results either from the increase of the number of crystals, $C^{(0)}$, or from the decrease of crystallized mass, $C^{(1)}$. As temperature drops, the nucleation rate drops according to Eq. (21), and more particles are generated per unit time, as given by Eq. (14). The crystal mass concentration, according to Eq. (15), grows more slowly because of the decrease of growth rate coefficient at low temperature.

The time evolution of nucleation rate [Fig. 1(c)] is caused by the decline of supersaturation due to crystalliza-

TABLE I. Parameter values in figures (unless otherwise noted in captions).

\begin{tabular}{ccccccccc}
\hline \hline$C_{0}^{(0)}$ & $C_{0}^{(1)}$ & $S_{0}$ & $J_{0}$ & $\lambda$ & $w$ & $\varepsilon$ & $h$ & $n$ \\
\hline 0 & 0 & 10 & $10^{6}$ & 0 & 2.0 & 1.0 & 100 & 20 \\
\hline \hline
\end{tabular}




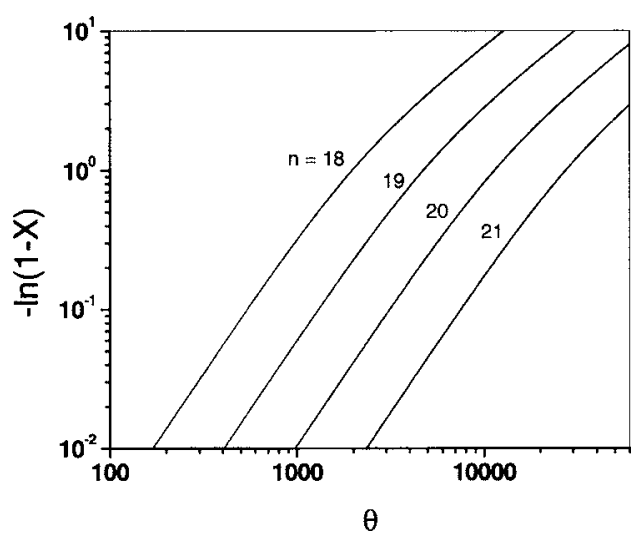

FIG. 2. Effect of temperature dependence of interfacial energy with $\Theta$ $=0.97$ and other parameters given in Table I.

tion. The nucleation rate drops quickly because of the sharp decrease of supersaturation. As the supersaturation decreases, the energy barrier for nucleation, $W^{*}$, tends to increase, thus the nucleation rate, according to Eq. (4), will decrease. The temperature effect on nucleation rate is also confirmed in Fig. 1(c). Initially a substantial nucleation rate decrease is observed when the temperature varies from 0.970 to 0.976 . Then nucleation is quenched at the end of crystallization as the supersaturation $S_{\text {sat }}$ approaches unity.

The characteristic Avrami plots are presented in Fig. $1(\mathrm{~d})$, and a larger crystallization rate is observed at lower temperature. The Avrami exponents at the four temperatures (the slopes of the characteristic Avrami plots) are around 1.95, independent of the temperature. The Avrami exponent is hardly affected by temperature near $T_{m}$.

The effect of $n$, the temperature power dependence for interfacial energy, is presented in Fig. 2. The temperature sensitivity of crystallization is strongly dependent on $n$. When $n$ is small, crystallization rate does not change much with temperature, thus large $n(\approx 20)$ is suggested here. According to Eqs. (3) and (6), increasing $n$ will raise the energy barrier for nucleation and thus decrease the nucleation rate. We observed a large difference in crystallization rate for a small difference in $n$, as shown in Fig. 2. We also find that $n$ has little influence on Avrami exponents.

The investigation of the activation energy in the growth

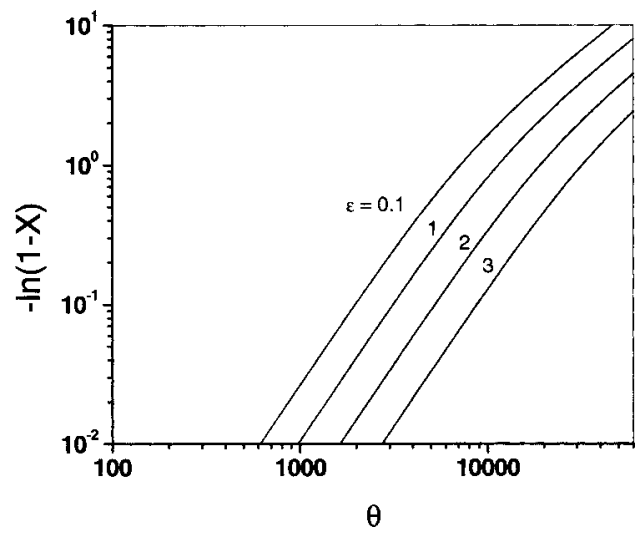

FIG. 3. Effect of activation energy $\varepsilon$ with $\Theta=0.97$ and other parameters given in Table I.

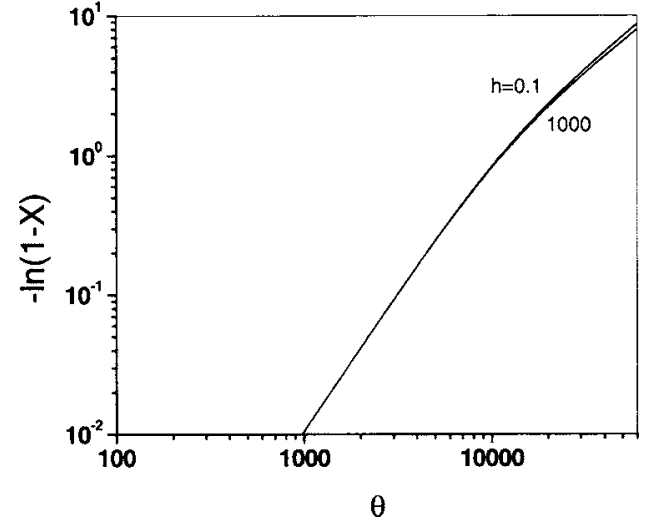

FIG. 4. Effect of phase transition enthalpy $h$ with $\Theta=0.97$ and other parameters given in Table I.

rate coefficient $k_{g}$ is presented in Fig. 3. The value of $\varepsilon$ is chosen to vary from 0.1 to 3 with $\Theta=0.97$ and other parameters given in Table I. No apparent slope difference is observed in the characteristic Avrami plots; thus, varying $\varepsilon$ does not influence the value of Avrami exponents. According to Eq. (8), the increase of $\varepsilon$ will lead to the decline of crystal growth rate coefficient, and a larger overall crystallization rate is indeed observed when $\varepsilon$ is small. Despite more than one order of magnitude change in the value of $\varepsilon$, however, the influence of this parameter on the time evolution of the crystallization is not as much as $n$.

The enthalpy of phase transition, $h$, is another parameter included in our distribution model for isothermal crystallization. According to experimental measurements, ${ }^{4,10} \Delta H$ is around $300 \mathrm{~kJ} / \mathrm{mol}$ for most polymers, so that $h$ is about 100. To explore the effect of phase transition enthalpy, we chose a wide range of $h$, varying from 0.1 to 1000, in Fig. 4 . We observe no kinetics contribution from the $h$ variation because $\exp (-h / \Theta)$ in Eqs. (13) and (15) is quite small and $S$ is the dominant term in the equations. Thus, the crystallization rate does not change much for large values of $h$.

The nucleation rate prefactor, $J_{0}$, according to Eq. (20) is related to interfacial energy, density, and polymer molecular weight; thus different nucleation rate prefactors are expected for different polymer systems. The investigation of the effect of nucleation prefactor is presented in Fig. 5. As is evident

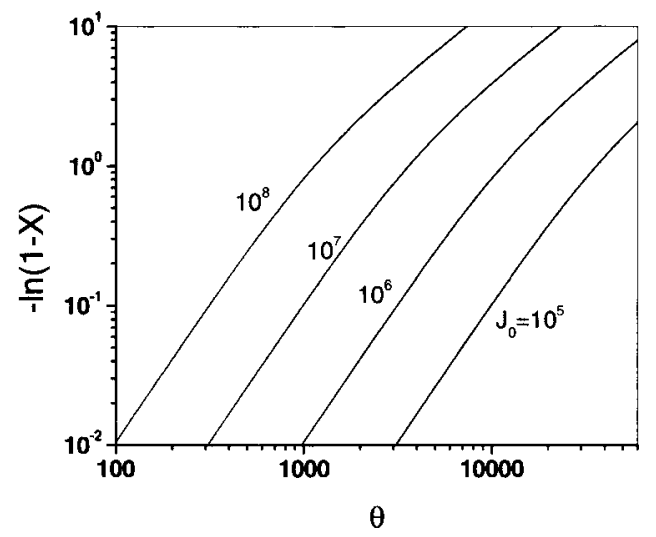

FIG. 5. Effect of nucleation rate prefactor $J_{0}$ with $\Theta=0.97$ and other parameters given in Table I. 


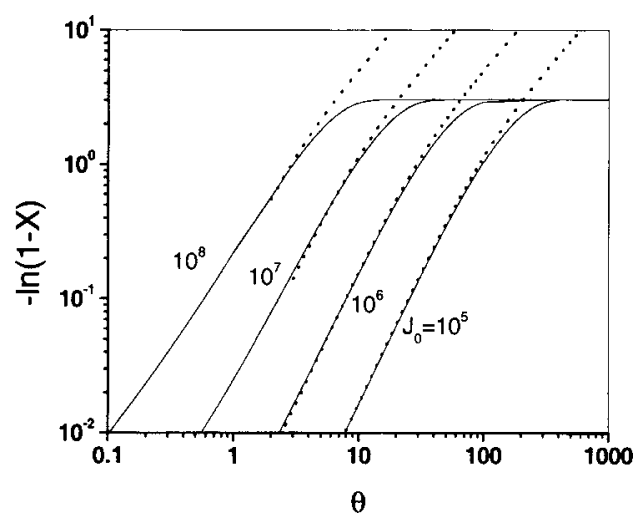

FIG. 6. Effect of denucleation at different nucleation rates with $\Theta=0.9$ and other parameters given in Table I. The dotted lines denote the moment solutions while the solid lines represent the numerical solutions.

from Eqs. (14)-(16), increasing $J_{0}$ speeds the growth rate and the onset of nucleation, resulting in a larger number of crystals. Shorter crystallization times are therefore required to reach the final state when the nucleation rate prefactor is large, as shown in Fig. 5. For Avrami plots, varying $J_{0}$ causes only a lateral shift in $\theta$. The nearly linear relationship between $\log [-\ln (1-X)]$ and $\log \theta$ allows only an order of magnitude lateral shift in $\theta$ as $J_{0}$ is increased by two orders of magnitude.

Denucleation, the reverse of nucleation, occurs when the crystal size is smaller than the critical crystal size. Denucleation has considerable influence on the characteristic Avrami plots, especially at the end of crystallization when denucleation dominates over nucleation. At the beginning of crystallization, most polymer crystals are larger than the critical crystal size, thus denucleation, compared with nucleation, is negligible. The critical crystal size, however, increases as the supersaturation $S_{\text {sat }}$ declines when polymer deposits on the crystal, thus more crystals become smaller than the critical size. These unstable crystals will dissolve rapidly. At the end of crystallization, denucleation and ripening become dominant because the supersaturation is close to unity, and consequently nucleation vanishes. Figure 6 presents the effect of denucleation by comparison of moment and numerical solutions at different nucleation rates. The dotted lines represent the characteristic Avrami plots of moment solutions, for which denucleation is not considered. The solid points are numerical solutions including denucleation, where numerical error tolerance is chosen to be 0.0001 in the calculation of $S$. At the beginning, the denucleation rate is small, and the moment solution agrees with the numerical solution very well. This is because the small denucleation rate delays the dominating influence of denucleation. ${ }^{28}$ As supersaturation decreases, the discrepancy between moment solution and numerical solution caused by denucleation becomes increasingly apparent. The numerically computed crystallinity reaches its asymptote while the crystallinity computed by the moment method continues to increase. The enthalpy of phase transition $h$ and interfacial energy $\omega$ also have an effect on denucleation. The critical crystal size is very small, according to Eq. (16), when $S$ is large and $w$ is small $(<0.1)$ or $h$ is large $(>100)$, thus most crystals are larger than the critical size and are stable. These crystals will keep growing instead of dissolving, so denucleation is negligible.

To explore the effect of the exponent of growth and dissociation rate, $\lambda$ is chosen to be $0,1 / 3$, and $2 / 3$, representing surface independent, diffusion controlled, and surface controlled deposition rates, respectively. Figure 7 presents the effect of $\lambda$ at different nucleation rates, varying from $10^{5}$ to $10^{8}$. The dotted lines are moment solutions $(\lambda=0)$. The solid
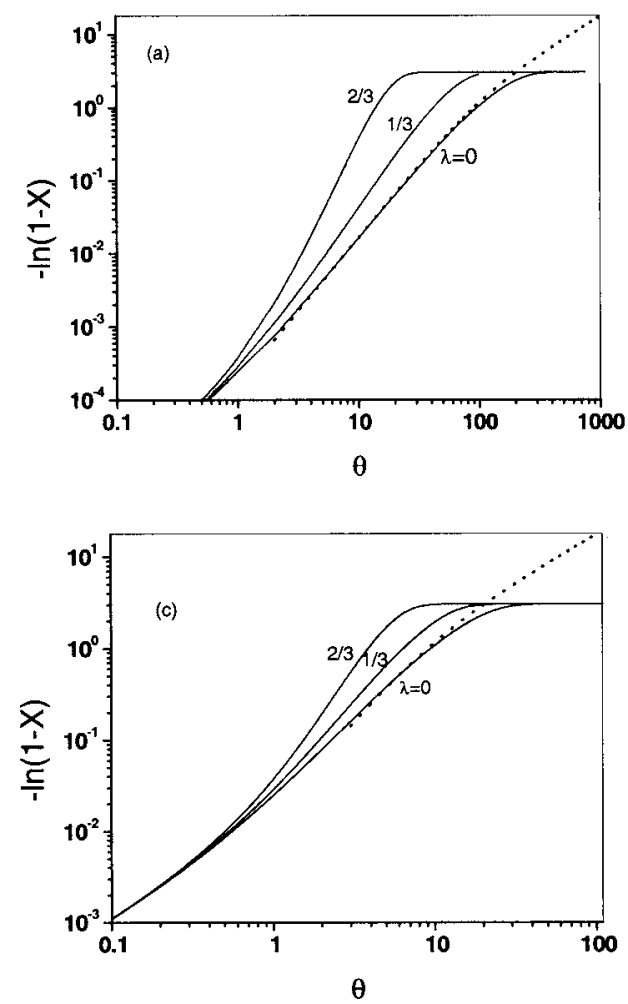
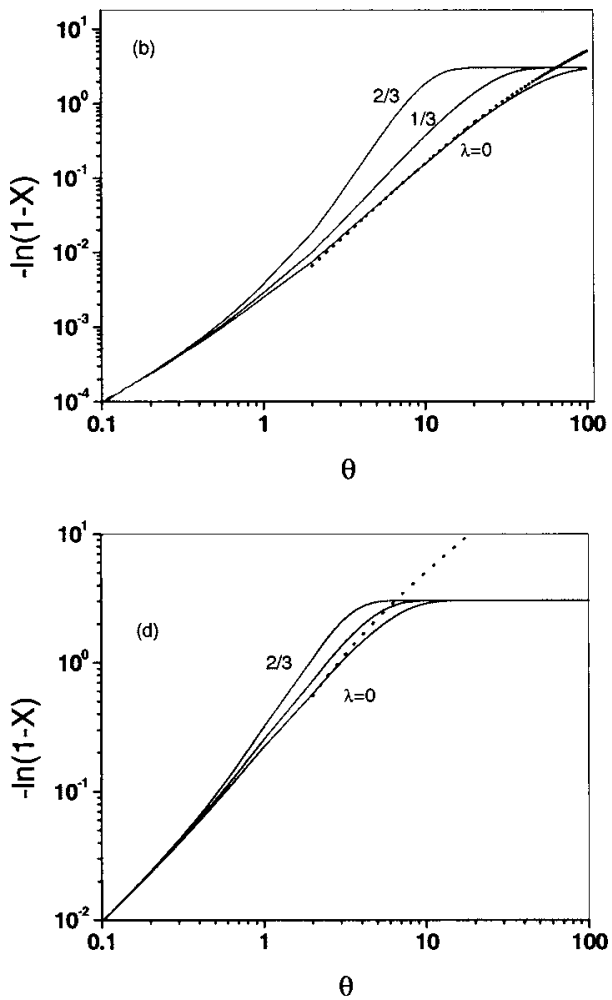

FIG. 7. The effect of $\lambda$ on the Avrami plots with $\Theta=0.9$ at different nucleation rate prefactors: (a) $J_{0}=10^{5}$, (b) $J_{0}=10^{6}$, (c) $J_{0}=10^{7}$, and (d) $J_{0}=10^{8}$ and other parameters given in Table I. The dotted lines denote the moment solutions while the solid lines represent the numerical solutions. 


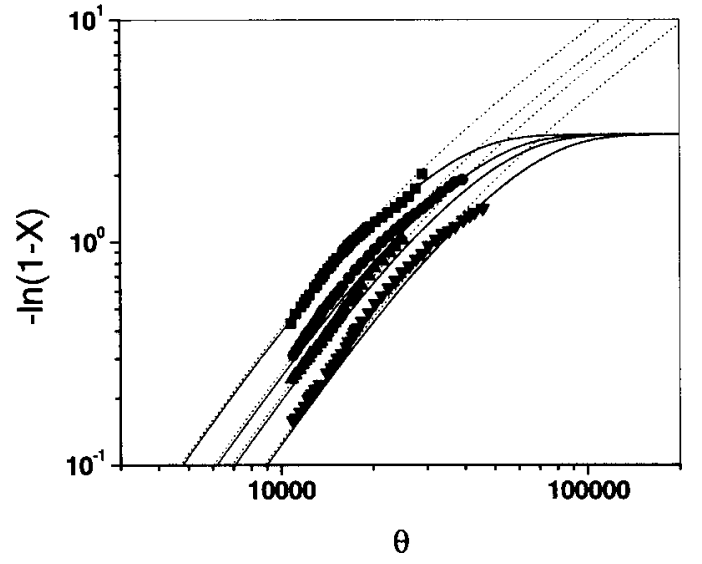

FIG. 8. The fit of distribution model to the experimental data for PBFP at four temperatures: ( $\mathbf{\square}) 229.8^{\circ} \mathrm{C},(\bullet) 231^{\circ} \mathrm{C},(\boldsymbol{\Delta}) 230.5^{\circ} \mathrm{C},(\boldsymbol{\nabla}) 231.5^{\circ} \mathrm{C}$, and other parameters given in Table I. The dotted lines denote the moment solutions while the solid lines represent the numerical solutions.

lines are the numerical solutions with $\lambda=0,1 / 3$, and $2 / 3$. The increase of $\lambda$, according to Eq. (8), leads to the increase of crystal growth rate coefficient, thus larger crystallization rate is observed when $\lambda=2 / 3$. Though larger crystallization rates are observed by a decrease of $\varepsilon$ or an increase of $J_{0}$, these parameters do not have a major influence on the Avrami exponent. Therefore, compared to the influence of other parameters, the Avrami exponent is critically dependent on $\lambda$.

The validity of the distribution kinetics model is also examined by comparison with experimental data (Fig. 8). Since the simulation results of moment methods are based on dimensionless time $\theta=t \gamma \mu_{\infty} x_{m}^{\lambda}$, a transposition of the original experimental data, based on real time, is applied to compare with computation results. According to the definition of dimensionless time, Eq. (11), a horizontal transposition of $\log \left(\gamma \mu_{\infty} x_{m}^{\lambda}\right)$ units is applied to the experimental measurements to convert the experimental data into plots based on dimensionless time $\theta$. The scattered points are experimental data $^{4}$ for poly[bis(trifluoroethoxy)phosphazene] (PBFP) based on scaled time $\theta$ at $T=229.8,230.5,231.0$, and $231.5^{\circ} \mathrm{C}$. The solid lines are the simulation results at these four temperatures. Good agreement between experimental data and simulation results is observed. The measured horizontal transposition is 2.34; thus $\gamma \mu_{\infty}=218.78 \mathrm{~s}^{-1}$ for the case $\lambda=0$. This is consistent with the understanding that $\gamma \mu_{\infty}$ is independent of temperature. The transposition therefore provides a value of the parameter $\gamma$. An apparent discrepancy is observed at the end of crystallization, where the experimental data slightly curve down from the model. This inconsistency is believed to result from the increasing effect of Ostwald ripening (denucleation) that cannot be incorporated in the moment equations. For the numerical solution represented by the solid line in Fig. 8, an apparent plateau is observed at the end of crystallization, evidence of the increasing effect of denucleation. Compared with the moment solution (dotted lines), a more quantitatively realistic description is achieved by numerical solution due to the inclusion of denucleation and ripening.

The moment method provides an easy solution in mod-

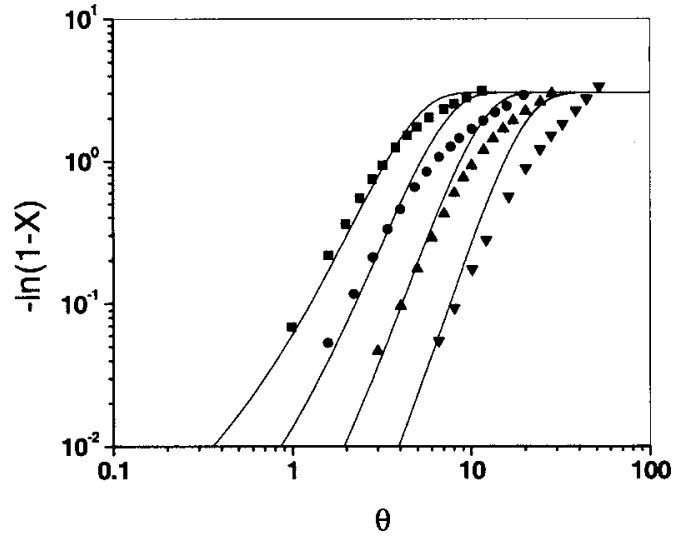

FIG. 9. The fit of distribution model with to the experimental data for PET at four temperatures: $(\boldsymbol{\square}) 185^{\circ} \mathrm{C},(\boldsymbol{O}) 195^{\circ} \mathrm{C},(\boldsymbol{\Delta}) 205^{\circ} \mathrm{C},(\boldsymbol{\nabla}) 215^{\circ} \mathrm{C}$, with $\lambda=2 / 3$, and other parameters given in Table I.

eling polymer crystallization with surface-independent crystal growth $(\lambda=0)$. When crystal growth rate is not surfaceindependent $(\lambda>0)$, however, the moment equations do not have a closed form, thus the numerical schemes must be applied, as shown in Eqs. (20) and (21). Figure 9 presents the fit of distribution model to the experimental data $^{27}$ for poly(ethyleneterephthalate-imide) (PET), which we suggest has a surface dependent crystal growth mechanism $(\lambda$ $=2 / 3$ ). By considering the denucleation effect, the numerical solution provides a more realistic prediction than the moment method at the end of crystallization. The measured horizontal transposition of experimental data is 1.30.

The value of $\lambda$ is determined by the crystal growth mechanism; surface independent $(\lambda=0)$, diffusion controlled $(\lambda=1 / 3)$, and surface dependent $(\lambda=2 / 3)$ crystal growth mechanisms were previously reported. ${ }^{18}$ These three well understood mechanisms provide good predictions to most experimental measurements. However, experiments with large Avrami exponents $(m>3)$, which cannot be explained by these three mechanisms, are also occasionally reported, ${ }^{8,9,24,29}$ thus it is necessary to propose a larger value $(\lambda>2 / 3)$. Figure 10 presents the fit of distribution model with $\lambda=1$ to the experimental measurements ${ }^{9}$ having a large Avrami exponent at three different temperatures. The points

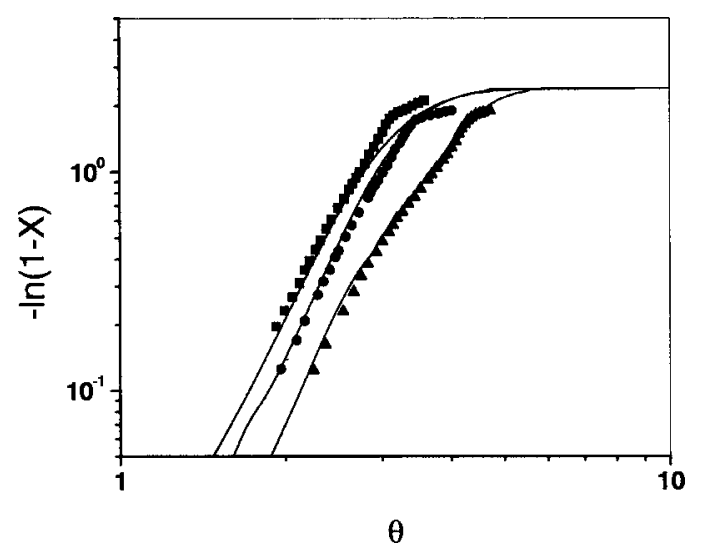

FIG. 10. The fit of distribution model with $J_{0}=10^{8}$ to the experimental data for PEO at three temperatures: (অ) $42{ }^{\circ} \mathrm{C},(\bullet) 43{ }^{\circ} \mathrm{C},(\boldsymbol{\Delta}) 44{ }^{\circ} \mathrm{C}, \lambda=1$, and other parameters given in Table I. 
represent the experimental data ${ }^{9}$ of poly(ethylene oxide) (PEO), where the measured Avrami exponent is around 4.5; the solid lines stand for the simulation results. By a horizontal transposition of 0.36 to the experimental measurements, the modeling result fits the experimental data rather well.

It is worthwhile to reemphasize why the computations represent the experimental data so well. Two features are particularly essential. First, a larger slope of the Avrami plot requires a larger exponent $\lambda(0<\lambda<1)$ in Eq. (8), indicating a stronger dependence of the growth and dissociation rate coefficients on cluster mass. Second, the high sensitivity of the crystallinity time dependence is due to the large value, $n \approx 20$, of the power in Eq. (6) for the interfacial energy. Finally, the placement of the crystallinity curves by transposing them on the time axis determines the coefficient $\gamma$ in Eq. (8). Thus the model neatly provides a systematic approach to representing and understanding the underlying phenomena of polymer crystallization.

\section{CONCLUSION}

Based on our earlier distribution kinetics model, ${ }^{1}$ we have investigated temperature effects in detail for 2D lamellar polymer crystallization. This model accounts for the overlapping of nucleation, crystal growth, and denucleation in polymer crystallization, processes which complicate the solidification of polymer solution or melt. Temperature affects these processes through kinetics and thermodynamics. We have suggested how our previous kinetics distribution model $^{1}$ can be applied to the investigation of isothermal crystallization. Proposing temperature dependences for interfacial energy, growth and dissociation rate coefficients, and equilibrium solubility, we studied crystallization behavior at different temperatures. This entailed the effects of growth rate activation energy, enthalpy of phase transition, nucleation rate prefactor, and the power dependence of interfacial energy on temperature. Computations (numerical solutions of the population balance equation) show that the Avrami exponent mainly depends on $\lambda$, the power dependence of growth rate on crystal mass. The characteristic Avrami plots of simulation results yielded very good fits to experimental measurements. This supports the assumed temperature dependence of interfacial energy, diffusion, growth rate coefficient, and equilibrium solubility. We also find that proposing a large $\lambda$ is the only way to predict polymer crystallization with large Avrami exponent (greater than 3). In addition, for interfacial energy a large value of the temperature power $n(\approx 20)$ is critical for explaining the temperature sensitivity of crystallization kinetics. Therefore, by appropriately choosing $\lambda$ and $n$, the cluster size distribution model is able to describe different types of polymer crystallization.

${ }^{1}$ J. Yang, B. J. McCoy, and G. Madras, J. Chem. Phys. 122, 64901 (2005).

${ }^{2}$ G. Madras and B. J. McCoy, Chem. Eng. Sci. 59, 2753 (2004).

${ }^{3}$ J. D. Robson, Acta Mater. 52, 4669 (2004).

${ }^{4}$ J. T. Xu, J. P. Fairclough, S. M. Mai, and A. J. Ryan, Macromolecules 35, 6937 (2002).

${ }^{5}$ S. O. Kim and I. J. Chung, Macromolecules 33, 7548 (2000).

${ }^{6}$ Y. He, B. Zhu, W. Kai, and Y. Inoue, Macromolecules 37, 8050 (2004).

${ }^{7}$ H. Hama and K. Tashiro, Polymer 44, 6973 (2004).

${ }^{8}$ P. Supaphol and J. E. Spruiell, J. Appl. Polym. Sci. 75, 44 (2000).

${ }^{9}$ Q. Guo, C. Harrats, G. Groeninckx, and M. H. J. Koch, Polymer 42, 4127 (2001).

${ }^{10}$ R. J. Ciora and J. H. Magill, Macromolecules 23, 2350 (1990).

${ }^{11}$ S. Acierno and N. Grizzuti, Macromolecules 35, 5043 (2002).

${ }^{12}$ J. K. Doye and D. Frenkel, J. Chem. Phys. 110, 2692 (1999).

${ }^{13}$ B. J. McCoy, J. Phys. Chem. Solids 63, 1967 (2004).

${ }^{14}$ J. Leisen and H. W. Beckham, Macromolecules 37, 8028 (2004).

${ }^{15}$ J. K. Doye and D. Frenkel, J. Chem. Phys. 109, 10033 (1998).

${ }^{16}$ R. B. McClurg and R. C. Flagan, J. Colloid Interface Sci. 228, 194 (1998).

${ }^{17}$ D. W. Oxtoby, J. Phys.: Condens. Matter 4, 7627 (1992).

${ }^{18}$ G. Madras and B. J. McCoy, Phys. Chem. Chem. Phys. 5, 5459 (2003).

${ }^{19}$ D. W. Oxtoby, J. Phys.: Condens. Matter 4, 7627 (1992).

${ }^{20}$ A. W. Adamson and A. P. Gast, Physical Chemistry of Surfaces, 6th ed. (Wiley-Interscience, New York, 1997), p. 328.

${ }^{21}$ P. G. Vekilov, Cryst. Growth Des. 4, 671 (2004).

${ }^{22}$ J. S. Rowlinson and B. Widom, Molecular Theory of Capillarity (Oxford University Press, New York, 1982), p. 249.

${ }^{23}$ B. J. McCoy, Ind. Eng. Chem. Res. 40, 5147 (2001).

${ }^{24}$ K. Nagarajan, K. Levon, and A. S. Myerson, J. Therm Anal. Calorim. 59, 497 (2000).

${ }^{25}$ G. Madras and B. J. McCoy, J. Chem. Phys. 115, 6699 (2001).

${ }^{26}$ G. Madras and B. J. McCoy, Acta Mater. 51, 2031 (2003).

${ }^{27}$ J. Xiao, H. Zhang, X. Wan, D. Zhang, Q. Zhou, E. M. Woo, and S. R. Turner, Polymer 43, 7377 (2002).

${ }^{28}$ G. Madras and B. J. McCoy, J. Chem. Phys. 117, 8042 (2002).

${ }^{29}$ J. A. Martins, W. Zhang, V. Carvalho, A. M. Brito, and F. O. Soares, Polymer 44, 8071 (2003). 